\title{
Sex-specific speed-accuracy tradeoffs shape neural processing of acoustic signals in a grasshopper \\ 3 Jan Clemens ${ }^{1}$, Bernhard Ronacher ${ }^{2}$, Michael S. Reichert ${ }^{3}$
}

4

Affiliations:

1 European Neuroscience Institute Göttingen - A Joint Initiative of the University Medical Center Göttingen and the Max-Planck Society, Grisebachstrasse 5, Göttingen, 37077, Germany 2 Behavioral Physiology Group, Department of Biology, Humboldt-Universität zu Berlin, Germany 3 Department of Integrative Biology, Oklahoma State University, Stillwater OK USA

Correspondence: clemensjan@gmail.com

Funding:

JC: DFG CL 596/1-1 (32951824), SPP 2205 (430158535)

BR: DFG Ro 547/12-1

MR: US National Science Foundation International Research Fellowship Program (IRFP 1158968)

Acknowledgments:

Michael Rumpold assisted with the experiments, and members of the Behavioral Physiology Group helped with field collection and husbandry.

Contributions:

Designed experiments and collected the behavioral data - BR, MR

Fitted and analyzed the model - JC

Wrote the manuscript - JC, BR, MR 


\begin{abstract}
Speed-accuracy tradeoffs - being fast at the risk of being wrong - are fundamental to many decisions and natural selection is expected to resolve these tradeoffs according to the costs and benefits of behavior. We here test the prediction that females and males should integrate information from courtship signals differently because they experience different payoffs along the speed-accuracy continuum. We fitted a neural model of decision making (a drift-diffusion model of integration to threshold) to behavioral data from the grasshopper Chorthippus biguttulus to determine the parameters of temporal integration of acoustic directional information used by male grasshoppers to locate receptive females. The model revealed that males had a low threshold for initiating a turning response, yet a large integration time constant enabled them to continue to gather information when cues were weak. This contrasts with parameters estimated for females of the same species when evaluating potential mates, in which response thresholds were much higher and behavior was strongly influenced by unattractive stimuli. Our results reveal differences in neural integration consistent with the sex-specific costs of mate search: Males often face competition and need to be fast, while females often pay high error costs and need to be deliberate.
\end{abstract}




\section{Introduction}

Sensory information is inherently noisy. Repeated sampling and integration of information over time reduces noise, and is a ubiquitous strategy in information processing and decision making [1-3]. The neural algorithm by which ecologically-relevant sensory information is integrated is expected to be shaped by a fundamental tradeoff between the speed of decision making and the accuracy of the inferred sensory input $[1,4,5]$. Studies of animals trained in artificial decisionmaking tasks show that the resolution of the speed-accuracy tradeoff depends on the relative costs of delaying the decision to accumulate more information compared to the costs of making an error based on insufficient information, the signal-to-noise ratio, and the stability of the sensory information over time [6-12]. However, all of these factors vary in natural environments, and among individuals, sexes and species [3,13,14]. While variation in speed-accuracy tradeoffs with condition or experience has been reported for naturalistic tasks [15-19], there exists little direct evidence that natural integration processes are shaped by selection, for instance from comparisons across groups expected to face different costs $[3,20]$.

The processing of signals related to mate choice presents a clear instance in which selection likely favors different resolutions of the speed-accuracy tradeoff in the two sexes, which in turn are expected to result in sex differences in temporal integration: Integration processes in males should facilitate the fast decisions required for successful competition, while integration in females should be slower, but enable more accurate decisions about male quality. The existence of sex-specific circuits in the nervous system suggests that temporal integration could indeed be implemented in a sex-specific manner [21,22], but whether the characteristics of temporal integration differ between males and females in a natural task is unclear. Here we combine an existing behavioral data set [23] with new data and fit a drift-diffusion model [5] to characterize how the nervous system accumulates sensory cues and triggers decisions in mate searching. Based on the expected costs and benefits of different integration strategies under sexual selection theory, we test predictions for how integration may differ between males and females evaluating acoustic signals of the opposite sex.

The grasshopper Chorthippus biguttulus provides an excellent model for studies of temporal integration because both males and females produce and respond to acoustic signals during mate searching (Fig. 1A) [24], but integration and decision making strategies are expected to differ between the sexes because they are subject to different selection pressures [25]. Males produce calling songs to find females; receptive females are stationary but respond with songs that facilitate mate localization by the male [26]. In $C$. biguttulus, females pay high costs from making errors [25,27,28], because they are egg-limited and mating with a male of another species or of low genetic quality produces no or low-quality offspring. In addition, singing exposes females to predators and parasitoids. Females should therefore avoid responding to the song from males of another species or of low genetic quality. By contrast, females do not face competition from other females and therefore are not under pressure to be fast. Females are therefore expected to favor accuracy over speed when evaluating the male song pattern. This was confirmed by a drift-diffusion model for temporal integration based on female response behavior $[29,30]$. The model parameters indicated that females integrate information across the entire calling song of a male with a high threshold for response and very high negative 
weighting of unattractive song components (i.e., those of heterospecific or malformed males). These integration parameters ensure the accurate detection of unsuitable males combined with slow behavioral responses to attractive males.

Here, we extend the modelling approach to male mate localization, which is predicted to have very different integration characteristics. Female density is low in the visually cluttered environment and females lack conspicuous visual characteristics or long-range chemical cues that would allow males to find them. Chance encounters are therefore rare and the female response song is often the only possibility for localizing receptive females [28,31]. Furthermore, the speed of approach is critical because females already engaged in close-range courtship with faster arriving males will not continue to advertise their position, preventing slower males from localizing those females. Thus, although males rarely directly interact with one another in physical competitions, they nevertheless face high levels of competition to rapidly localize responsive females in a crowded and noisy environment [32]. Males should therefore favor speed over accuracy to a greater degree than females, although we do not expect males to completely disfavor accuracy when integrating directional cues from the female song, since localization errors increase the time exposed to predators and parasitoids, and will prevent them from finding the female. We predict therefore that males will have higher sensory weightings (or equivalently, a lower response threshold) than females, reflecting their speedier response. This puts them at risk of making errors if early sensory information is wrong. Furthermore, to maintain accuracy when directional cues are equivocal, we predict that integration times will be at least as long as typical female songs so that males can maximize the chances of integrating sufficient directional information from female signals.

To test these predictions, we used new and previously published behavioral data from a twospeaker playback design that measured male localization of artificial female songs with conflicting directional cues [23]. We applied a drift-diffusion model to the behavioral data to determine the parameters of temporal integration in males. The drift-diffusion model corresponded very well with males' decisions, and the model's parameters matched our predictions of long temporal integration times and a low threshold for response, which contrasts with the parameters determined for female behavior using the same model. This reveals sexspecific differences in the neural processing of sexual signals consistent with predictions from sexual selection theory. 


\section{Methods} Animals

Behavioral data were collected as described in [23]. We used lab-reared and wild-caught males of the species $C$. biguttulus. Lab-reared males were the F1 offspring from wild-caught individuals and were isolated by sex at the last instar nymph stage and reared in cages separated by sex. Wild-caught males could have mated previously but were kept separate from females for at least three days before the experiments, which is sufficient for them to regain motivation to respond to female signals $[33,34]$. To further minimize variation in male motivation to respond, we only tested males that responded to a test signal from an attractive female, indicating high motivation to engage in courtship behavior. We did not systematically track male age or exclude males based on their age. Both lab-reared and wild-caught males were grouphoused in male-only cages in similar conditions. In group housing, all males would have been exposed to the song of other males, as well as the songs of female playbacks used to assess male motivation and identify test subjects. We, as well as previous studies [35], did not observe any systematic differences in the selectivity of lab-reared or wild-caught males, nor any effect of previous experience on the integration of acoustic cues from females. There is also no evidence for learning in this communication system.

\section{Behavioral experiments}

Motivated adult were placed between two speakers that broadcast an artificial female song stimulus (Fig. 1B). A female song consists of subunits ("syllables") that are separated by pauses. The syllables in our female model song were separated by a $17.5 \mathrm{~ms}$ pause; each syllable consisted of 6 sound pulses (average pulse duration $10.7 \mathrm{~ms}$ ). This stimulus pattern was highly attractive and reliably elicited turning responses in males, allowing us to assess how directional cues from the stimulus were integrated by the males. Syllables that lack a pause or do not consist of distinct sound pulses are not attractive to males and fail to elicit male turning responses [36-38]. Individual syllables were manipulated to have timing or level differences between the speakers (see below). Stimuli were broadcast at $60 \mathrm{~dB}$ SPL at the position of the male. Males were presented with ten repetitions of each stimulus. We tested a total of 204 males and most males were tested with more than one stimulus. The median number of different stimuli tested per male (with 10 repetitions per stimulus) was 8 (inter-quartile range 510). Stimuli were repeated at a variable rate because each time the male moved, we had to reposition the speakers to center the male once he was again stationary. 44 stimuli contained 12 syllables to mimic a typical female song, but we also tested shortened stimuli with 8 (3 stimuli), 5 (33 stimuli) or 3 ( 1 stimulus) syllables to better characterize the dynamics of integration. We include data for male responses from a total of 81 stimuli (Fig. S1). Data for 38/81 stimuli were previously published in [23].

Male lateralization behavior was quantified as follows. First, the response for each male was quantified as the proportion of turns directed towards the stimulus channel designated as the reference out of the total number of turns towards either stimulus ("0" if the male turned away from the reference speaker, "1" when the male turned towards the reference speaker). For some stimuli, males responded to the stimulus but turned forward instead of towards one of the speakers and we scored these responses as " 0.5 ", equivalent to a decision probability of 0.5 towards (score 1.0) and 0.5 away (score 0.0 ) from the reference speaker. We then averaged the 
responses across all males tested with that stimulus ( $N=15-23$ males tested per stimulus, median 20 males). The experimental setup did not allow us to score turning latencies and those data were therefore not available for model fitting. However, our stimulus design, with conflicting cues placed in different positions within the song, allows us to reliably infer the dynamics of cue integration from the response scores (see below).

\section{Stimulus design}

The dynamics of sensory integration in males were inferred using artificial female songs that varied in duration and in the sequence of directional cues (see Fig. S1 for all stimulus patterns). Each syllable provided one of three types of directional cue: 1) Level cues: we generated stimuli with level differences by silencing some syllables on one speaker channel. This effectively results in an $8 \mathrm{~dB}$ interaural level difference [39,40], 2) Timing cues, in which the syllable from one speaker led the other by $4 \mathrm{~ms}$, or 3 ) no directional cues ("neutral"), in which syllables were presented simultaneously at equal amplitude from both channels. Both timing and level cues elicit orientation responses in male grasshoppers, but are expected to provide directional cues of different strength depending on the magnitude of each cue. We did not attempt to equalize the strength of the timing and level cues used for our stimuli, and instead estimated these parameters from the models. We systematically varied the number and location within the song of these directional cues to generate stimuli with different amounts of directional information, and in some cases with conflicting directional information. This stimulus design with serially conflicting directional cues was critical for calibrating the model parameters [41]. For instance, responses to songs in which syllables at the beginning of the song indicated a female in the direction of one speaker and those at the end of the song indicated a female in the opposite direction reveal over how many syllables males integrate and when decisions are fixed. Combining this stimulus design with a neural model of decision making (see below) allowed us to infer the sensory weights and thresholds, and estimate decision times, even in the absence of reaction time data. A control stimulus with neutral directional cues elicited turning responses with random directions (score 0.53 , random turning would produce 0.5 ). Another control stimulus that was broadcast from only one speaker, reliably elicited turns in males $(90 \%$ of trials) and all of those turns were correctly directed towards the broadcasting speaker. See Fig. $\mathrm{S} 1$ for a list of all stimulus patterns used in this study.

\section{Comparison of correlations between the stimulus and the behavior}

We assessed the strength of the relationship (squared Pearson's $r$ ) between the males' turning responses and different parts of the 12 syllable stimuli, by dividing each stimulus in thirds (syllables 1-4, 5-8, 9-12), calculating the average directional cue for each third, and correlating that average with the males' turning responses (Fig. S2B). To account for stimulus-intrinsic correlations, arising from regularity in the stimulus sequences (Fig. S1), we also correlated the average cue for each third with the average cue over the full song (Fig. S2A). This revealed that the middle of the song was most strongly correlated with the cues from the full song, which is a result of our stimulus design because the cue direction often changed halfway through the song (Fig. S1). The difference of the correlation obtained from the behavior and from the full stimulus indicates stimulus thirds that are more or less influential on the behavior than expected from the stimulus statistics (Fig. S2C, 1C). 


\section{Modelling}

The stimulus is defined as a sequence $s(t)$ with $t=[1, \ldots, T]$, $T$ being the number of syllables in the stimulus, and a sign indicating the side of the cue relative to the reference speaker ("-1" away from the reference, " +1 " towards the reference, " 0 " neutral).

\section{Averaging model}

As a baseline, the averaging model simply averages the directional information over the full song and the predicted response $\rho$ is then a function of that average: $\rho=f(x(T+1))$, with $x(t+1)=$ $x(t)+Z(s(t)), x(0)=0$. The sign function $Z(s(t))$ returns -1 if $s(t)<0$ and +1 otherwise. To account for saturation effects, we set $f$ to be a sigmoidal, which was fitted to minimize the mean squared error over all stimuli between the prediction $\rho$ of the averaging model and the males' turning response $r$. However, this only marginally increased the performance of the simple averaging models ( $r^{2}$ linear: $0.72, r^{2}$ sigmoidal: 0.75 ).

\section{Drift-diffusion model}

In a drift-diffusion model, the cues from each syllable are weighted and assigned a sign based on the direction they indicate. The weighted cues are then integrated with an integration timescale $\mathrm{T}$, which determines the "leakiness" of integration, with a value of infinity corresponding to perfect integration with no forgotten information, and smaller values corresponding to forgetting of information that came before that time interval. Noise $\sigma$ is added to the integrated sensory information from each syllable, and the decision is fixed when a decision threshold of either $+\theta$ or $-\theta$ is crossed, indicating the decision to turn towards or away from the reference speaker, respectively. If the threshold is not crossed before the end of the song, the decision is made based on the sign of the integrated information at the end of the song. An urgency gain parameter was included to account for the possibility that sensory weights increase or decrease over time [6,42]; increased urgency may be expected for males that need to localize receptive females quickly upon receipt of evidence that one is present. More precisely, the integrated information $\mathrm{x}$ after syllable $\mathrm{t}$ is given by

$$
x(t+1)=\left\{\begin{array}{l}
-\theta, \text { if } x(t)<-\theta \\
\theta, \text { if } x(t)>\theta \\
x(t) / \tau+w(t) s(t)+\eta(t) \sigma, \text { otherwise }
\end{array}\right.
$$

with $x(0)=0$, an integration time constant $\mathrm{T}$, and a decision threshold $\theta$. Noise $\eta(t)$ was drawn at each time step from a normal distribution with zero mean and unit variance. For timing cues, $w$ was fixed to 1.0 for all models. For level cues, $w$ was fixed to 1.0 for models that did not differentially weight timing and level cues ("single cue" in Table S1) and optimized during fitting for models that did ("two cues"). For models with urgency gain, the sensory weight changed over time and was defined as $w(t)=w^{*}(1+(t-1) y)$, with $y$ being the urgency gain [42]. Experiments with alternative implementations in which the urgency gain reduced the threshold over time [6] yielded similar results of negligible gain. The decision threshold $\theta$ was sticky - once it was crossed, integration ceased and $x(t)$ was fixed to $\pm \theta$. The predicted response, $\rho$, was determined by the sign of the integrated information after the last syllable, $Z(x(T+1))$ averaged over 1000 different instantiations of the noise $\eta$. The simple averaging model can be considered a special case of a drift-diffusion model with $\mathrm{w}=1$ for level and timing cues, $\sigma=0, \mathrm{~T}=\infty$, and $\theta=\infty$. 


\section{Model fitting and evaluation}

The parameters of the drift-diffusion models were optimized by minimizing the mean-squared error between the predicted and the males' responses using a Genetic Algorithm ([43], see [44] for details). To speed up convergence, upper and lower bounds were defined for all parameters: $0<\mathrm{W}_{\mathrm{L}}<10,1<\mathrm{T}<40,0<\sigma<5,0<\theta<10,0<\mathrm{Y}<10$. We ensured that these bounds did not affect the final parameter estimates. Fits were evaluated using leave-one-out cross-validation. That is, the model parameters were fitted on all but one stimulus (and its mirror version) and a prediction was then generated for the left-out stimuli. Doing this for all stimuli resulted in 81 parameter estimates and 157 predictions. The squared Pearson's coefficient of correlation, $r^{2}$, between the predictions and the males' responses was used to quantify model performance. Different models were compared using Akaike's Information Criterion (AIC), which penalizes models with many parameters. The $A I C$ score is given by $A I C=2 k+n \ln (e)$, where $k$ is the number of parameters of the model, $n$ is the number of samples used for fitting the model, and $e$ is the sum of squared residuals between the predicted and the male's responses: $e=\sum(r-\rho)^{2}$. Smaller AIC scores are better. 


\section{Results}

\section{Noisy integration to threshold explains turning behavior.}

Based on the correlation of the cues in different parts of the song with behavior, we find that the beginning of the song influences behavior more than expected (Fig. 1C, S2). This suggests an integration process in males that does not always consider information from the full female song but instead fixes decisions rapidly and dynamically with the available sensory evidence [23]. To account for this finding, we fitted different models of cue integration and decision making. Model comparison (Akaike Information Criterion) revealed that the simplest model that explained our data is a drift-diffusion model with an infinite integration time in which timing and level cues had different weights, but their weights did not change over time (i.e., an urgency gain of zero) (Table S1, Fig. 2A-C, Fig. S3). We consider this as the best fit model in discussions below. A threshold-less model that simply averaged directional cues with identical weights for both cue types across the entire song performed worse (Fig. 2C) as did a drift-diffusion model variant with identical weights for both cue types (Table S1). These simpler models performed well on average (Table S1) because for many stimuli in our dataset, the average cue still predicted the behavior well. However, the performance gap between these models and our best fit model was much higher for stimuli with conflicting or mixed cues, for which correct weighting and stopping of integration after threshold-crossing were crucial model parameters for predicting males' behavior (Fig. 2C). Adding even more complexity to the model with the addition of leaky integration or an urgency parameter did not improve performance (Table S1). Model parameters were similar for all of the fitted variants of the drift-diffusion model, indicating that our results are robust to changes in model complexity.
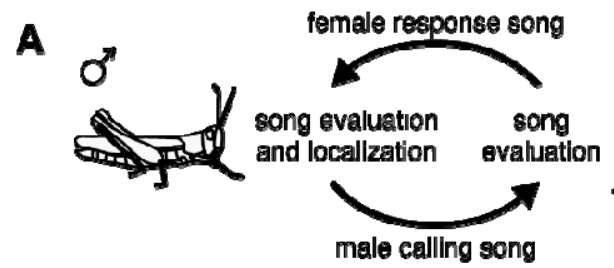

B directional cues
from female song

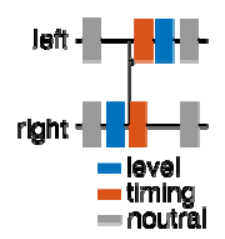

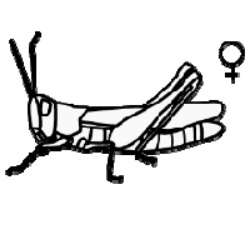

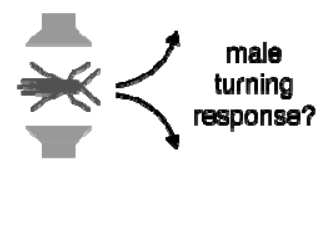

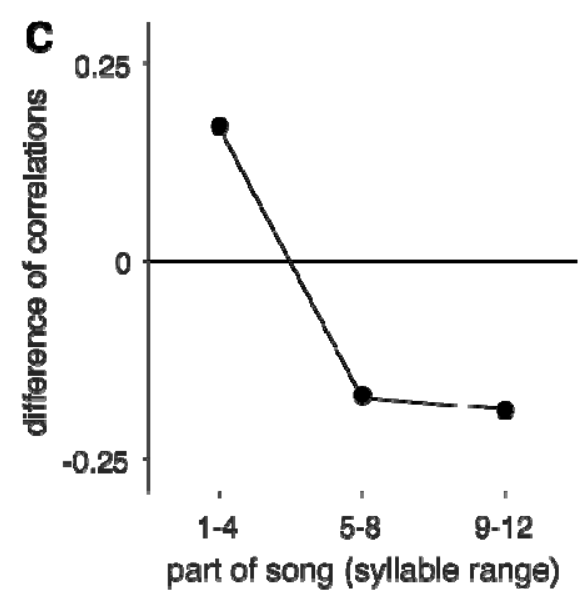

Figure 1 - Performance of a simple averaging model compared to male behavior

A Bidirectional acoustic communication during mate search in the grasshopper $C$. biguttulus.

B Schematic of the paradigm - two speakers were placed on either side of the male, artificial female song is played, and the direction of the male turning response is scored. Directional cues are provided by each syllable and arise from level differences (sound on one speaker only, blue) or timing differences (sound on one speaker delayed by $4 \mathrm{~ms}$, red). Both cue types are known to elicit turning responses in males.

C Difference in the correlation of different thirds of the 12 syllable songs observed in behavior and estimated from the stimulus statistics. The beginning tends to be more, the middle and end less influential on behavior than expected from the stimulus statistics. See Fig. S2 for details and number of stimuli. 

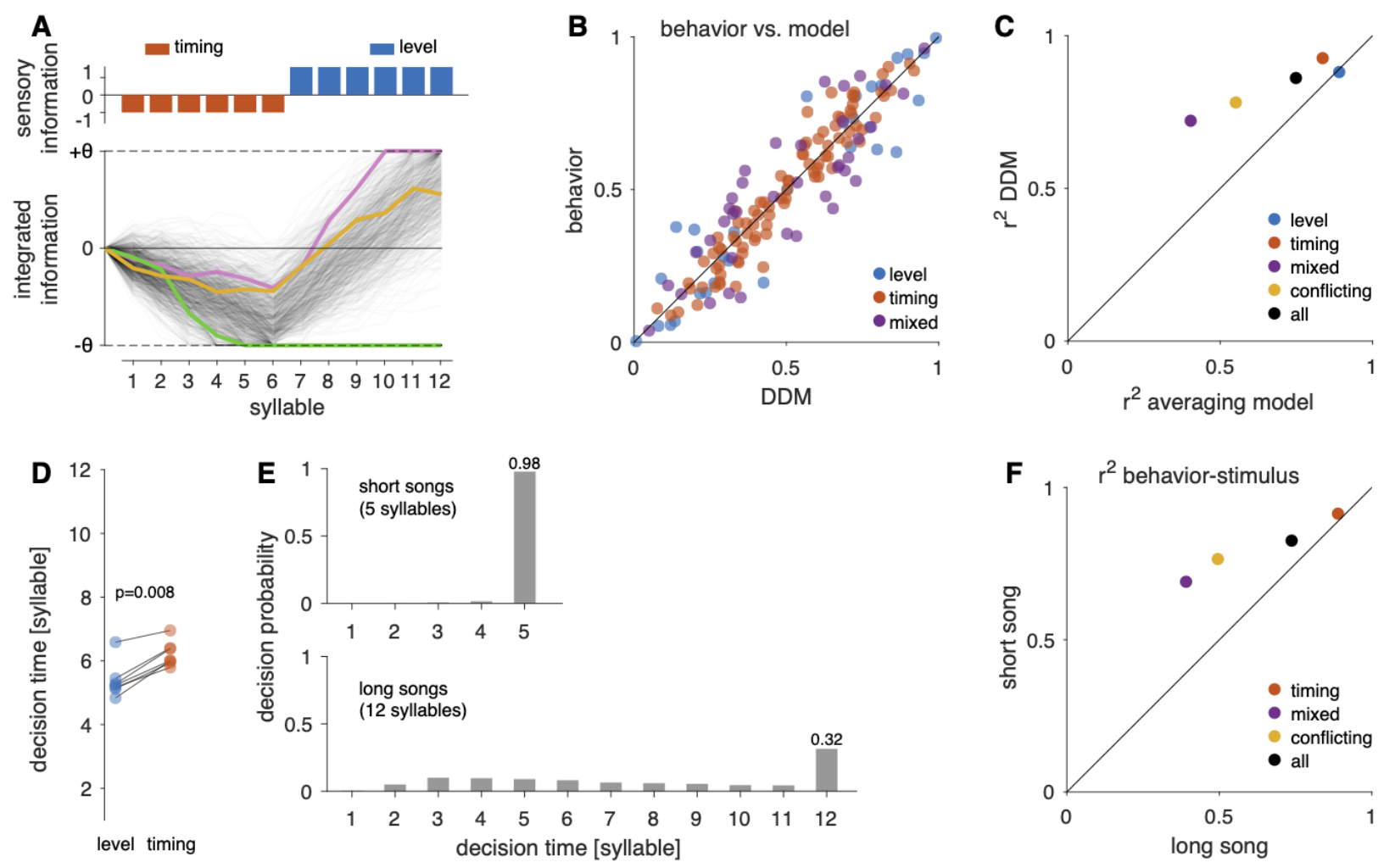

Figure 2 - A drift-diffusion model (DDM) reproduces the behavior well and reveals dynamics of temporal integration.

A DDM responses for a 12-syllable stimulus (top) with the first 6 syllables containing timing cues (red) away from the reference speaker (-), and 6 syllables with level cues (blue) towards the reference speaker (+). Each cue type is assigned a weight (height of bars, top). Stimulus information is integrated noisily and a decision towards the reference (+) or opposite (-) speaker is fixed when the decision threshold $\theta$ is crossed. Thin black lines indicate 1000 runs with independent noise realizations. Colored lines highlight example runs that cross the negative threshold (green), the positive threshold (purple), or no threshold (orange), in which case the decision is determined by the sign of the evidence at song end (+).

B Proportion of turns towards the reference speaker in model and behavior. Color indicates cue composition of the songs. Diagonal line corresponds to perfect match between model and behavior. All points are close to that line $\left(r^{2}=0.86\right)$.

C $\mathrm{R}^{2}$ between model predictions and behavioral data for the best fitting model (DDM) compared to that of a simple averaging model for different data subsets (see legend). The best fitting model outperforms the simple averaging model in particular for stimuli with mixed (purple, stimuli containing timing and level cues) and conflicting cues (yellow, stimuli with cues from both sides).

D Mean decision time (syllable at which threshold is crossed) for seven stimuli with matching patterns (lines) but level (blue) or timing (red) cues. Consistent with their higher weight in the model, level cues drive decisions by about 1 syllable earlier ( $p=0.008$, left-sided sign test). See Fig. S4 for the decision time distributions for each of the stimuli depicted here.

E Decision times for short songs with 5 syllables (top, $\mathrm{N}=66$ stimuli) and long songs with 12 syllables (bottom, N=83 stimuli). For most long songs, integration reaches threshold before song end. For nearly all short songs, integration fails to cross threshold. Short song mostly contained timing cues (Fig. S1). Numbers in the last bar indicate the probability of not reaching the threshold for the two stimulus sets.

F Correlation of behavior with the average directional cue over the full song for short and long songs. The failure of threshold crossing before song end for short songs ( $E$, top) leads to integration over the full song and a higher correlation with the average directional cue. There is no "level" stimulus set for this analysis since our data set did not contain such stimuli for short songs (cf. C, Fig. S1). 
The best fit model indicated that males can integrate directional cues over the whole song, and even in models with a leaky integration, the time constant was estimated at 24 syllables (Table $\mathrm{S} 1$ ), which is twice as long as both the longest song in our dataset (12 syllables; $1.19 \mathrm{~s}$ ) and a typical female song (12-15 syllables, mean $\pm S D=1.18 \pm 0.23 \mathrm{~s}$; [45]). Thus, sensory information from the whole song has the potential to influence the localization response. The decision threshold $\theta$ of the best fit model had a value of 7.14. Level and timing cues were weighted differently, with the level cue outweighing the timing cue by a factor of 1.65 . The minimum number of syllables required to cross the threshold $(\theta / w)$ was therefore 8 for timing and 5 for level cues, meaning that the stronger level cues drove faster decisions (Figs. 2D, S4). The low threshold resulted in decisions usually being fixed before the end of long 12-syllable songs (Fig. $2 \mathrm{E})$, consistent with our finding that the beginning of the song is more and the end of the song is less influential than expected for observed male turning responses (Figs. 1C, S2). This means that males trade accuracy in favor of speed, since responding before the end of song can result in localization errors if sensory information early in the song is unreliable. For the short songs, which largely consisted of the weaker timing cues, sensory information was insufficient to drive decisions by crossing the threshold in our model, and the turning direction was determined by the value of the integrated information at the song end (Fig. 2E). This is consistent with the observation that the average directional cue over the full song is more predictive of behavior for the short songs, than for the long songs (Fig. 2F). The noise level $\sigma$ of the best fit model was 2.25; thus, the signal-to-noise ratios $(w / \sigma)$ were 0.44 for timing cues and 0.73 for level cues. Localization cues provided by single syllables are therefore relatively noisy, and integration is indeed necessary to infer sound direction reliably. 


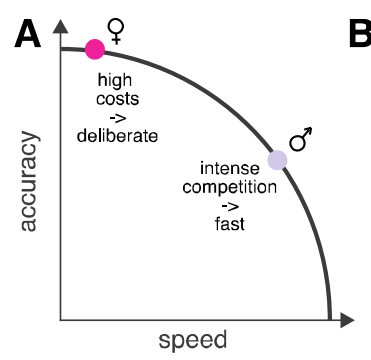

357

358

359

360

361

362

363

364

365

366

367

368

369

370

371

372

373

374

375

376

377

378

379

380

381

382

383

384

385

\section{Discussion}
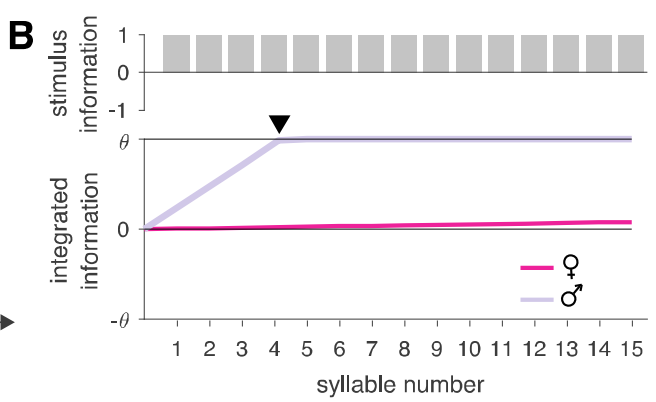

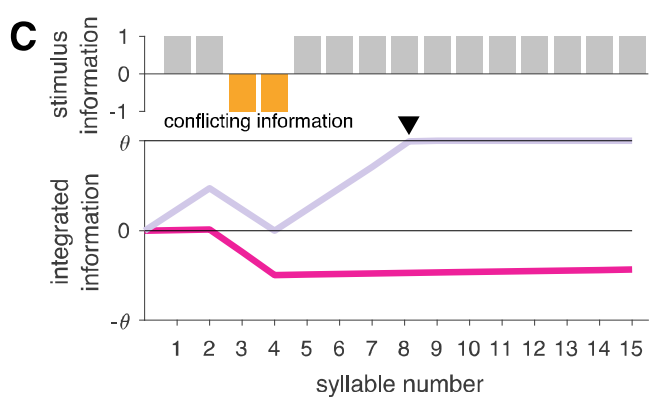

Figure 3 - Sex-specific speed-accuracy trade-offs arise from differential integration dynamics.

A Males and females differentially resolve speed-accuracy trade-offs when responding to acoustic communication signals. Females (magenta) pay high costs from errors and therefore maximize their accuracy by deliberation at the cost of speed. Males (gray) face intense competition with other males and trade accuracy in favor of speed. B, C Integration dynamics tune decision making to the sex-specific speed-accuracy trade-offs. Shown is the integrated information (bottom) for females (magenta) and males (gray) for stimuli (top) with unequivocal (B) and conflicting $(\mathrm{C})$ information. Integrated information is scaled relative to the decision thresholds $\theta$ to facilitate the comparison between sexes. For females, individual stimulus elements correspond to species-typical (gray) and untypical (orange) patterns. Species-typical cues have low weight and are not sufficient to fix decisions before song end (B). Conflicting (negative) cues have a strong weight and practically veto positive responses (C). For males, the stimuli correspond to directional cues. Individual cues have high weight, which accelerates decisions (black arrowhead) for unequivocal information (B). Long integration times improve accuracy when cues are conflicting (C). See also Table S2. Female data from [29].

Our drift-diffusion model of temporal integration applied to male behavior demonstrates that sexual selection has shaped the neural processing of acoustic stimuli to favor speedy decisions in males, in contrast to the slower, but more accurate decisions in females (Fig. 3A). The model accurately reproduced the males' localization behavior (Fig. 2B, C) and the model parameters describe an integration process that is consistent with the pressures facing males to rapidly localize a stationary, singing female in a noisy environment (Fig. 2D-F, Table S1). The same modeling technique was previously used on females of the same species evaluating songs of potential mates [29], and the differences in model parameters correspond with expectations of sexual selection theory that females should have a higher threshold for response and strongly avoid unattractive signal characteristics (Table S2). This is a rare demonstration of variation in temporal integration strategies associated with ecologically relevant and natural behaviors.

\section{Decisions in males are fast for strong cues and accurate for weak cues}

We found that males had a low threshold for response: the average time to decision inferred from the model was much less than the duration of the standard female song stimulus used in this study (Fig. 2D, E). Thus, when the evidence is strong, males can decide quickly. This corresponds with the behavior of males in localization experiments, in which they frequently turn towards a song before it ends [40]. The best fit model had higher weights for level cues than for timing cues (Table S2, Fig. S3B), and decisions were therefore faster with level cues than with timing cues (Fig. 2D). This does not imply that level cues always predominate over timing cues; instead the difference likely arose because of the specific values chosen for each cue: the 
unilateral level cues (resulting in an approximately $8 \mathrm{~dB}$ ILD) were expected to provide stronger directional information than the bilateral timing cues (4 ms ITD) [40]. Importantly, this finding implies that cues are weighted by their strength, such that decision making is accelerated when evidence is strong.

Although we expected males' decisions to be biased towards speed, accuracy is also important because mistakes in localization could cause males to move out of hearing range of the female and prevent them from finding one another $[35,46]$. The signal-to-noise ratio for a single syllable was low ( 0.44 and 0.73 , respectively), and males therefore did integrate over multiple syllables, although they usually reached threshold and made a decision before they heard all of the syllables in the full song (Fig. 2D, E). This also means that males would be less accurate in case directional cues early in the song indicate the wrong direction. Nevertheless, the model indicated that males had the capacity to integrate over a much longer time period if directional cues were weak and the threshold was not reached (Table S1). Thus, when directional cues were too weak for a speedy decision, males could integrate additional sensory information, which should improve signal-to-noise ratios and ultimately lateralization accuracy [32]. This explains the high accuracy of male directional responses in the presence of noise [35]. Long integration times are maladaptive when the information being integrated changes more rapidly than the integration time constant, leading to erroneous decisions [10,47]. However, in this system long integration may have few costs because the information evaluated by males in the female song, her position, is constant prior to the turning decision because females remain stationary while singing. The integration dynamics in males therefore resolve the speedaccuracy tradeoff by allowing for flexibility in decision making: Sensory information is able to drive fast responses when it is strong, but long integration times allow accurate localization of the female in case of weak cues.

While our experiments were designed to assess the decision-making strategies of males on a population level, some variation in decision-making strategies could depend on male state or consistent differences between individuals. In other species, competitively inferior males use socalled satellite or sneaker strategies to avoid direct competition with dominant males [48]. However, in $C$. biguttulus direct agonistic interactions between males are rare and the primary means of competition is the ability to rapidly localize females. Slow decision making, similar to females', is therefore unlikely to be an advantageous alternative strategy for males in this species. Given that our model explains the behavior measured from different sets of males so well $\left(r^{2}=0.86\right.$, Fig. $\left.2 \mathrm{~B}, \mathrm{C}\right)$, variation among individuals is likely low, and our main conclusion that males trade accuracy in favor of speed - is likely to be robust to these factors.

\section{Integration of courtship signals is tuned to sex-specific costs}

Our finding that male $C$. biguttulus have a low threshold for response contrasts with the results from previous studies using a similar behavioral and modeling paradigm to characterize temporal integration in females of the same species $[29,30]$. Females were tested with songs consisting of a mixture of attractive and unattractive syllables. There was a large difference between males and females in how they weighted sensory information. In females, positive cues had a weak influence and on their own could not reach the threshold by the end of the 
song; in other words, females rarely commit to a positive decision before the end of the song. However, negative cues (i.e., unattractive song syllables) had a much stronger weight and even a few unattractive syllables could reach the threshold for not responding. In contrast, in males, we found that clear directional information had a strong weight and was capable of driving responses before the end of a typical female song. Both sexes had integration times that were longer than the duration of typical songs, but in females this likely serves less to enhance the signal-to-noise ratio (as we argue is the case for males), but rather to ensure the detection of unattractive elements at any point in the song, preventing them from initiating courtship with a low-quality or heterospecific male.

There are some differences in the behavioral paradigms because females were tested for a response to songs with both positive and negative information on male attractiveness, while males were tested using only attractive syllables but with varied directional cues. Pattern and directional information are extracted from the song in parallel pathways and the pattern decision then gates turning [36]. Thus, turning in males in this study reflects both the attractiveness of the song syllable and the quality of directional cues. Despite these differences, both the female decision to respond and the male decision to turn signal readiness of each sex to further escalate the courtship interaction. Therefore, the integration differences between males and females reflect differences in the costs and benefits of decision-making strategies affecting each sex. Future studies examining integration of stimuli with unattractive pattern information in males would further elucidate sex differences in temporal processing in this species.

The neural circuits that integrate directional cues over time to control male turning behavior are unknown. Peripheral circuits extract directional cues from afferent inputs but do not integrate this information across multiple syllables [49-51]. The evaluation of the song pattern and integration of directional cues is likely to happen in the brain and its results are relayed to the motor centers via descending interneurons [52], but this has not been assessed systematically. In the female brain, auditory activity has been recorded in the lateral protocerebrum, the superior medial protocerebrum and the central complex (CX) $[53,54]$ and electrical stimulation of the $\mathrm{CX}$ can elicit the behavioral responses to song in females [55]. In the insect brain, the $\mathrm{CX}$ is a central circuit for orientation behavior with integrator properties [56,57]. It may therefore drive responses also in males and CX neurons themselves or their presynaptic partners may have sex-specific properties that reflect the sex-specific speed-accuracy trade-offs evident from behavior.

Although the specific neural circuits have not been identified, our drift-diffusion model is realistic because it replicates identified neural processes. All model parameters map to biophysical properties of decision making neurons and circuits [58,59]: Sensory weights could correspond to the number and strength of synapses to an integrating neuron. The integration time constant could correspond for instance to the kinetics of intracellular calcium, or to factors that determine the dynamics of a recurrent network [60,61]. The decision threshold could correspond to a spiking threshold determined by the density of sodium channels at the spike initiation zone or controlled by neuromodulators $[60,62,63]$. Sexual selection could act on these parameters to produce the sex-specific integration of sensory information seen in grasshoppers. Our results 
bioRxiv preprint doi: https://doi.org/10.1101/2020.07.20.212431; this version posted January 20, 2021. The copyright holder for this preprint (which was not certified by peer review) is the author/funder, who has granted bioRxiv a license to display the preprint in perpetuity. It is made available under aCC-BY-NC 4.0 International license.

483 therefore point the way towards a study of the evolution of sensory processing mechanisms in 484 realistic ecological contexts and natural behaviors.

485 


\section{References}

1. Gold, J. I. I. \& Shadlen, M. N. 2007 The Neural Basis of Decision Making. Annu. Rev. Neurosci. 30, 535-574. (doi:10.1146/annurev.neuro.29.051605.113038)

2. Heitz, R. P. 2014 The speed-accuracy tradeoff: history, physiology, methodology, and behavior. Frontiers in Neuroscience 8, 865. (doi:10.3389/fnins.2014.00150)

3. Chittka, L., Skorupski, P. \& Raine, N. E. 2009 Speed-accuracy tradeoffs in animal decision making. Trends in Ecology \& Evolution 24, 400-407. (doi:10.1016/j.tree.2009.02.010)

4. Reddi, B. A. \& Carpenter, R. H. 2000 The influence of urgency on decision time. Nature Neuroscience 3, 827830. (doi:10.1038/77739)

5. Ratcliff, R. \& Mckoon, G. 2008 The diffusion decision model: theory and data for two-choice decision tasks. Neural computation 20(4), 873-922. (doi:10.1162/neco.2008.12-06-420)

6. Tajima, S., Drugowitsch, J. \& Pouget, A. 2016 Optimal policy for value-based decision-making. Nat Commun 7, 12400. (doi:10.1038/ncomms12400)

7. Palmer, J., Huk, A. C. \& Shadlen, M. N. 2005 The effect of stimulus strength on the speed and accuracy of a perceptual decision. Journal of Vision 5, 1-1. (doi:10.1167/5.5.1)

8. Zylberberg, A., Fetsch, C. R., Shadlen, M. N. \& Frank, M. J. 2016 The influence of evidence volatility on choice, reaction time and confidence in a perceptual decision. eLife 5, e17688. (doi:10.7554/eLife.17688)

9. Davidson, J. D. \& El-Hady, A. 2019 Foraging as an evidence accumulation process. PLoS Comput Biol 15, e1007060. (doi:10.1371/journal.pcbi.1007060)

10. Piet, A. T., El-Hady, A. \& Brody, C. D. 2018 Rats adopt the optimal timescale for evidence integration in a dynamic environment. Nat Commun 9, 1-12. (doi:10.1038/s41467-018-06561-y)

11. Constantinople, C. M., Piet, A. T. \& Brody, C. D. 2019 An Analysis of Decision under Risk in Rats. Current Biology 29, 2066-2074.e5. (doi:10.1016/j.cub.2019.05.013)

12. Zhang, S. X., Miner, L. E., Boutros, C. L., Rogulja, D. \& Crickmore, M. A. 2018 Motivation, Perception, and Chance Converge to Make a Binary Decision. Neuron 99, 376-388. (doi:10.1016/j.neuron.2018.06.014)

13. Sponberg, S., Dyhr, J. P., Hall, R. W. \& Daniel, T. L. 2015 Luminance-dependent visual processing enables moth flight in low light. Science 348, 1245-1248. (doi:10.1126/science.aaa3042)

14. Stöckl, A. L., Kihlström, K., Chandler, S. \& Sponberg, S. 2017 Comparative system identification of flower tracking performance in three hawkmoth species reveals adaptations for dim light vision. Philosophical Transactions of the Royal Society B: Biological Sciences 372, 20160078. (doi:10.1098/rstb.2016.0078)

15. Chittka, L., Dyer, A. G., Bock, F. \& Dornhaus, A. 2003 Bees trade off foraging speed for accuracy. Nature 424, 388-388. (doi:10.1038/424388a)

16. Wang, M.-Y., Brennan, C. H., Lachlan, R. F. \& Chittka, L. 2015 Speed-accuracy trade-offs and individually consistent decision making by individuals and dyads of zebrafish in a colour discrimination task. Animal Behaviour 103, 277-283. (doi:10.1016/j.anbehav.2015.01.022)

17. Franks, N. R., Dornhaus, A., Fitzsimmons, J. P. \& Stevens, M. 2003 Speed versus accuracy in collective decision making. Proceedings of the Royal Society of London. Series B: Biological Sciences 270, 2457-2463.

(doi:10.1098/rspb.2003.2527)

18. Bonachea, L. A. \& Ryan, M. J. 2011 Localization Error and Search Costs during Mate Choice in Túngara Frogs, Physalaemus pustulosus. Ethology 117, 56-62. (doi:10.1111/j.1439-0310.2010.01843.x) 
19. Ings, T. C. \& Chittka, L. 2008 Speed-Accuracy Tradeoffs and False Alarms in Bee Responses to Cryptic Predators. Current Biology 18, 1520-1524. (doi:10.1016/j.cub.2008.07.074)

20. El-Hady, A., Davidson, J. D. \& Gordon, D. M. 2019 Editorial: An Ecological Perspective on Decision-Making: Empirical and Theoretical Studies in Natural and Natural-Like Environments. Front. Ecol. Evol. 7, 712. (doi:10.3389/fevo.2019.00461)

21. Dickson, B. J. 2008 Wired for sex: the neurobiology of Drosophila mating decisions. Science (New York, N.Y.) 322, 904-909. (doi:10.1126/science.1159276)

22. Yang, C. F. \& Shah, N. M. 2014 Representing Sex in the Brain, One Module at a Time. Neuron 82, $261-278$. (doi:10.1016/j.neuron.2014.03.029)

23. Reichert, M. S. \& Ronacher, B. 2019 Temporal integration of conflicting directional cues in sound localization. J. Exp. Biol. 222(23), jeb.208751. (doi:10.1242/jeb.208751)

24. Helversen, von, D. 1972 Gesang des Männchens und Lautschema des Weibchens bei der Feldheuschrecke Chorthippus biguttulus (Orthoptera, Acrididae). Journal of Comparative Physiology: Neuroethology, Sensory, Neural, and Behavioral Physiology 81, 381-422. (doi:10.1007/BF00697757)

25. Helversen, von, O. \& Helversen, von, D. 1994 Forces driving coevolution of song and song recognition in grasshoppers. 253-284. In: Schildberger K., Elsner N. (eds) Neural basis of behavioural adaptations. Fischer, Stuttgart.

26. Helversen, von, D. 1997 Acoustic communication and orientation in grasshoppers. 301-341. In: Lehrer M. (ed.) Orientation and communication in arthroprods. Birkhäuser, Basel.

27. Kuijper, B., Pen, I. \& Weissing, F. J. 2012 A Guide to Sexual Selection Theory. Annu. Rev. Ecol. Evol. Syst. 43, 287-311. (doi:10.1146/annurev-ecolsys-110411-160245)

28. Kriegbaum, H. 1989 Female choice in the grasshopper Chorthippus biguttulus. Naturwissenschaften 76, 81-82. (doi:10.1007/BF00396715)

29. Clemens, J., Krämer, S. \& Ronacher, B. 2014 Asymmetrical integration of sensory information during mating decisions in grasshoppers. Proc Natl Acad Sci U S A 111, 16562-16567. (doi:10.1073/pnas.1412741111)

30. Clemens, J., Aufderheide, J. \& Ronacher, B. 2017 Relative weighting of acoustic information during mating decisions in grasshoppers indicates signatures of sexual selection. J Comp Physiol A 203, 891-901. (doi:10.1007/s00359-017-1200-x)

31. Kriegbaum, H. \& Helversen, von, O. 1992 Influence of Male Songs on Female Mating Behavior in the Grasshopper Chorthippus biguttulus (Orthoptera: Acrididae). Ethology 91, 248-254. (doi:10.1111/j.14390310.1992.tb00866.x)

32. Römer, H. 2013 Masking by Noise in Acoustic Insects: Problems and Solutions. 33-63. In: Brumm, H. (ed.) Animal Communication and Noise. Springer, Berlin.

33. Wirmer, A., Faustmann, M. \& Heinrich, R. 2010 Reproductive behaviour of female Chorthippus biguttulus grasshoppers. Journal of Insect Physiology 56, 745-753. (doi:10.1016/j.jinsphys.2010.01.006)

34. Heinrich, R., Kunst, M. \& Wirmer, A. 2012 Reproduction-Related Sound Production of Grasshoppers Regulated by Internal State and Actual Sensory Environment. Frontiers in Neuroscience 6. (doi:10.3389/fnins.2012.00089)

35. Reichert, M. S. 2015 Effects of noise on sound localization in male grasshoppers, Chorthippus biguttulus. Animal Behaviour 103, 125-135. (doi:10.1016/j.anbehav.2015.02.020)

36. Helversen, von, D. \& Helversen, von, O. 1995 Acoustic pattern recognition and orientation in orthopteran insects: parallel or serial processing? Journal of Comparative Physiology A: Neuroethology, Sensory, Neural, and Behavioral Physiology 177, 767-774. (doi:10.1007/BF00187635) 
37. Helversen, von, D. \& Helversen, von, O. 1997 Recognition of sex in the acoustic communication of the grasshopper Chorthippus biguttulus (Orthoptera, Acrididae). Journal of Comparative Physiology A: Neuroethology, Sensory, Neural, and Behavioral Physiology 180, 373-386. (doi:10.1007/s003590050056)

38. Ronacher, B. \& Krahe, R. 1998 Song recognition in the grasshopper Chorthippus biguttulus is not impaired by shortening song signals: implications for neuronal encoding. J Comp Physiol A 183, 729-735. (doi:10.1007/s003590050295)

39. Wolf, H. 1986 Response patterns of two auditory interneurons in a freely moving grasshopper (Chorthippus biguttulus L.). Journal of Comparative Physiology A: Neuroethology, Sensory, Neural, and Behavioral Physiology 158, 689-696. (doi:10.1007/BF00603826)

40. Helversen, von, D. \& Rheinlaender, J. 1988 Interaural intensity and time discrimination in an unrestraint grasshopper: a tentative behavioural approach. J Comp Physiol A 162, 333-340. (doi:10.1007/BF00606121)

41. Stine, G. M., Zylberberg, A., Ditterich, J. \& Shadlen, M. N. 2020 Differentiating between integration and nonintegration strategies in perceptual decision making. eLife 9, 8213. (doi:10.7554/eLife.55365)

42. Cisek, P., Puskas, G. A. \& El-Murr, S. 2009 Decisions in changing conditions: the urgency-gating model. Journal of Neuroscience 29, 11560-11571. (doi:10.1523/JNEUROSCI.1844-09.2009)

43. Mitchell, M. 1998 An Introduction to Genetic Algorithms (Complex Adaptive Systems). Third Printing. A Bradford Book.

44. Clemens, J. \& Ronacher, B. 2013 Feature extraction and integration underlying perceptual decision making during courtship behavior. Journal of Neuroscience 33, 12136-12145. (doi:10.1523/JNEUROSCI.0724-13.2013)

45. Helversen, von, D. \& Helversen, von, O. 1975 Verhaltensgenetische Untersuchungen am akustischen Kommunikationssystem der Feldheuschrecken (Orthoptera, Acrididae). J Comp Physiol 104, 301-323. (doi:10.1007/BF01379054)

46. Michelsen, A. \& Rohrseitz, K. 1997 Sound localisation in a habitat: an analytical approach to quantifying the degradation of directional cues. Bioacoustics 7, 291-313. (doi:10.1080/09524622.1997.9753341)

47. Dunlap, A. S., McLinn, C. M., MacCormick, H. A., Scott, M. E. \& Kerr, B. 2009 Why some memories do not last a lifetime: dynamic long-term retrieval in changing environments. Behavioral Ecology 20, 1096-1105. (doi:10.1093/beheco/arp102)

48. Kodric-Brown, A. 1986 Satellites and sneakers: opportunistic male breeding tactics in pupfish ( Cyprinodon pecosensis ). Behavioral Ecology and Sociobiology 19, 425-432.

49. Stumpner, A., Ronacher, B. \& Helversen, von, O. 1991 Auditory Interneurones in the Metathoracic Ganglion of the Grasshopper Chorthippus Biguttulus: II. Processing of Temporal Patterns of the Song of the Male. J. Exp. Biol. 158, 411-430.

50. Stumpner, A. \& Ronacher, B. 1991 Auditory Interneurones in the Metathoracic Ganglion of the Grasshopper Chorthippus Biguttulus: I. Morphological and Physiological Characterization. J. Exp. Biol. 158, 391-410.

51. Stumpner, A. \& Ronacher, B. 1994 Neurophysiological Aspects of Song Pattern Recognition and Sound Localization in Grasshoppers. Amer. Zool. 34, 696-705.

52. Ronacher, B., Helversen, von, D. \& Helversen, von, O. 1986 Routes and stations in the processing of auditory directional information in the CNS of a grasshopper, as revealed by surgical experiments. Journal of Comparative Physiology A: Neuroethology, Sensory, Neural, and Behavioral Physiology 158, 363-374. (doi:10.1007/BF00603620)

53. Kutzki, O. 2012 Kodierung verhaltensrelevanter Gesangsparameter bei Chorthippus biguttulus. PhD Thesis, Humboldt-Universität zu Berlin, Mathematisch-Naturwissenschaftliche Fakultät I. (doi:10.18452/16520) 
54. Bhavsar, M. B., Heinrich, R. \& Stumpner, A. 2015 Multielectrode recordings from auditory neurons in the brain of a small grasshopper. - PubMed - NCBI. Journal of Neuroscience Methods 256, 63-73. (doi:10.1016/j.jneumeth.2015.08.024)

55. Bhavsar, M.B., Stumpner, A. \& Heinrich, R. 2017 Brain regions for sound processing and song release in a small grasshopper. Journal of Insect Physiology 99, 15-24.

56. Pfeiffer, K. \& Homberg, U. 2014 Organization and functional roles of the central complex in the insect brain. Annual Review of Entomology 59, 165-184. (doi:10.1146/annurev-ento-011613-162031)

57. Seelig, J. D. \& Jayaraman, V. 2015 Neural dynamics for landmark orientation and angular path integration. Nature 521, 186-191. (doi:10.1038/nature14446)

58. Brody, C. D. \& Hanks, T. D. 2016 Neural underpinnings of the evidence accumulator. Current Opinion in Neurobiology 37, 149-157. (doi:10.1016/j.conb.2016.01.003)

59. O'Connell, R. G., Shadlen, M. N., Wong-Lin, K. \& Kelly, S. P. 2018 Bridging Neural and Computational Viewpoints on Perceptual Decision-Making. Trends in Neurosciences 41, 838-852. (doi:10.1016/j.tins.2018.06.005)

60. Zhang, S. X., Rogulja, D. \& Crickmore, M. A. 2019 Recurrent Circuitry Sustains Drosophila Courtship Drive While Priming Itself for Satiety. Current Biology 29, 3216-3228. (doi:10.1016/j.cub.2019.08.015)

61. Deutsch, D. et al. 2020 The Neural Basis for a Persistent Internal State in Drosophila Females. bioRxiv 1, 2020.02.13.947952. (doi:10.1101/2020.02.13.947952)

62. Marder, E. \& Thirumalai, V. 2002 Cellular, synaptic and network effects of neuromodulation. Neural Networks 15, 479-493.

63. Bargmann, C. I. 2012 Beyond the connectome: How neuromodulators shape neural circuits. Bioessays 34, 458465. (doi:10.1002/bies.201100185) 